\title{
Entropy and the Uncertainty Principle
}

\author{
Rupert L. Frank and Elliott H. Lieb
}

\begin{abstract}
We generalize, improve and unify theorems of Rumin, and Maassen-Uffink about classical entropies associated with quantum density matrices. These theorems refer to the classical entropies of the diagonals of a density matrix in two different bases. Thus, they provide a kind of uncertainty principle. Our inequalities are sharp because they are exact in the high-temperature or semi-classical limit.
\end{abstract}

\section{Introduction}

The von Neumann entropy of a quantum state (density matrix) can be calculated either in momentum space or in configuration space and the two are equal and non-negative. They can even be zero. Nevertheless, the corresponding classical entropies, determined by the diagonals of the two representations of the density matrix, can be different, and they can even be negative, but their sum cannot be arbitrarily small. This sum of the classical entropies can thus serve as a measure of the quantum mechanical uncertainty principle.

This point of view was advocated by Deutsch [3], who, among other things, proved a lower bound on this sum, which was later improved by Maassen and Uffink [8], following a conjecture of Kraus [6]. These inequalities were obtained for a general pair of bases, not just momentum and configuration space. In the momentum-configuration bases, an improvement on these previous inequalities was made by Rumin [9], who was able to add a term to the inequality involving the largest eigenvalue of the density matrix. He raised the question whether this additional term could be further improved by using a larger quantity, namely, the von Neumann entropy of the density matrix. In this paper, we prove that this surmise is correct.

Copyright (C) 2012 by the authors. This paper may be reproduced, in its entirety, for noncommercial purposes.

The work was partially supported by NSF Grants PHY-1068285 (R.L.F.) and PHY-0965859 (E.H.L.). 
We prove even more by combining the Maassen-Uffink investigation with the Rumin surmise. Rumin was concerned with the momentumconfiguration space duality, whereas Maassen-Uffink were concerned with arbitrary pairs of bases of the Hilbert space. For this, they introduced a parameter $c$ which somehow quantifies the disparity between the two bases. As one might expect, the $k, x$ pair has the largest $c$ value, i.e., $c=1$. We show how our theorem applies to any pair with the corresponding $c$-dependent improvement found in [8].

Our theorem and simple proof are supported by a semi-classical intuition, as evidenced by our use of the Golden-Thompson inequality. The only other ingredient in our proof is the Gibbs variational principle. Because our constant in Theorem 2.1 agrees with the semi-classical limit, it is the best possible.

\section{Rumin's Conjecture and Its Generalizations}

For any trace class operator $\gamma \geq 0$ on $L^{2}\left(\mathbb{R}^{d}\right)$, we denote by $\rho_{\gamma}(x)=\gamma(x, x)$ its density; see (2.2) for a precise definition. Moreover, its Fourier transform is

$$
\hat{\gamma}\left(k, k^{\prime}\right)=\iint_{\mathbb{R}^{d} \times \mathbb{R}^{d}} e^{2 \pi i\left(k \cdot x-k^{\prime} \cdot x^{\prime}\right)} \gamma\left(x, x^{\prime}\right) \mathrm{d} x \mathrm{~d} x^{\prime}
$$

and

$$
\rho_{\widehat{\gamma}}(k)=\hat{\gamma}(k, k)=\iint_{\mathbb{R}^{d} \times \mathbb{R}^{d}} e^{2 \pi i k \cdot\left(x-x^{\prime}\right)} \gamma\left(x, x^{\prime}\right) \mathrm{d} x \mathrm{~d} x^{\prime} .
$$

We note that if $\operatorname{Tr} \gamma=1$, then

$$
\int_{\mathbb{R}^{d}} \rho_{\gamma}(x) \mathrm{d} x=\int_{\mathbb{R}^{d}} \rho_{\widehat{\gamma}}(k) \mathrm{d} k=1 .
$$

Our main result is

Theorem 2.1. For any $\gamma \geq 0$ with $\operatorname{Tr} \gamma=1$ and

$$
\int_{\mathbb{R}^{d}} \rho_{\gamma}(x) \ln _{+} \rho_{\gamma}(x) \mathrm{d} x<\infty \quad \text { and } \int_{\mathbb{R}^{d}} \rho_{\widehat{\gamma}}(k) \ln _{+} \rho_{\widehat{\gamma}}(k) \mathrm{d} k<\infty,
$$

where $\ln _{+} \rho=\max \{\ln \rho, 0\}$, one has

$$
-\int_{\mathbb{R}^{d}} \rho_{\gamma}(x) \ln \rho_{\gamma}(x) \mathrm{d} x-\int_{\mathbb{R}^{d}} \rho_{\widehat{\gamma}}(k) \ln \rho_{\widehat{\gamma}}(k) \mathrm{d} k \geq-\operatorname{Tr} \gamma \ln \gamma .
$$

Remarks. (1) While the entropy on the right side of (2.1) is necessarily nonnegative, those on the left side can have either sign.

(2) Inequality (2.1) is saturated in the semi-classical limit. This can be verified by taking $\gamma=Z_{\beta}^{-1} \exp \left(-\beta\left(-\Delta+x^{2}\right)\right)$ and letting $\beta \rightarrow 0$ (see [9]).

(3) For $\gamma$ of rank one, this is Hirschman's inequality [5]. This was improved by Beckner [1]. Because of (2), however, this improvement is not possible if one allows for mixed states (i.e., $\gamma$ of higher rank). 
(4) The inequality for $\gamma$ equal to a multiple of a projection was proved in [9]. More generally, Rumin proves (2.1) with $\ln \|\gamma\|_{\infty}$ instead of $\operatorname{Tr} \gamma \ln \gamma$.

(5) The inequality shares the following tensorization property: If $d=n+m$, we can think of $L^{2}\left(\mathbb{R}^{d}\right)$ as $L^{2}\left(\mathbb{R}^{n}\right) \otimes L^{2}\left(\mathbb{R}^{m}\right)$. Then, the main inequality for $\gamma=\gamma_{n} \otimes \gamma_{m}$ equals the sum of the inequalities for $\gamma_{n}$ and $\gamma_{m}$.

(6) If, instead, we define the Fourier transform by

$$
\widetilde{\gamma}(p, q)=\iint e^{i(p \cdot x-q \cdot y)} \gamma(x, y) \frac{\mathrm{d} x \mathrm{~d} y}{(2 \pi)^{d}}
$$

and

$$
\rho_{\widetilde{\gamma}}(p)=\iint e^{i p \cdot(x-y)} \gamma(x, y) \frac{\mathrm{d} x \mathrm{~d} y}{(2 \pi)^{d}},
$$

then (2.1) becomes

$-\int \rho_{\gamma}(x) \ln \rho_{\gamma}(x) \mathrm{d} x-\int \rho_{\widetilde{\gamma}}(p) \ln \rho_{\widetilde{\gamma}}(p) \mathrm{d} p \geq-\operatorname{Tr} \gamma \ln \gamma+d \ln (2 \pi)$.

Theorem 2.1 is a special case of a more general Theorem 2.2 below. We listed Theorem 2.1 separately because it was the starting point of our investigation and was conjectured by Rumin.

The more general theorem includes the discrete case as well as the continuous case in Theorem 2.1. It is not entirely a triviality that the discrete and continuous cases are contained in one theorem because, as is well known, many entropy inequalities are true in one case and not in the other. For example, the discrete entropy is always positive while the continuous entropy can be, and often is, negative.

The general set-up consists of two sigma-finite measure spaces $(X, \mu)$ and $(Y, \nu)$. We denote by $L^{2}(X)$ and $L^{2}(Y)$ the corresponding spaces of squareintegrable functions. Let $\gamma$ be a non-negative operator on $L^{2}(X)$ with $\operatorname{Tr} \gamma=1$. Then, we have $\gamma=\sum_{j} \lambda_{j}\left|f_{j}\right\rangle\left\langle f_{j}\right|$ with orthonormal functions $f_{j}$ and numbers $\lambda_{j} \in[0,1]$ satisfying $\sum_{j} \lambda_{j}=1$. We define the density $\rho_{\gamma}$ of $\gamma$, a function on $X$, by

$$
\rho_{\gamma}(x)=\sum_{j} \lambda_{j}\left|f_{j}(x)\right|^{2} .
$$

By monotone convergence, we have

$$
\int_{X} \rho_{\gamma}(x) \mathrm{d} \mu(x)=\sum_{j} \lambda_{j}=\operatorname{Tr} \gamma=1 .
$$

Assume now that there is a unitary operator $\mathcal{U}: L^{2}(X) \rightarrow L^{2}(Y)$. For $\gamma$ as before, we define an operator $\hat{\gamma}$ on $L^{2}(Y)$ by

$$
\hat{\gamma}=\mathcal{U} \gamma \mathcal{U}^{*} \text {. }
$$

This operator is non-negative and has $\operatorname{Tr} \hat{\gamma}=1$. Its density $\rho_{\widehat{\gamma}}$ is defined similarly to that of $\rho_{\gamma}$, namely,

$$
\rho_{\widehat{\gamma}}(y)=\sum_{j} \lambda_{j}\left|g_{j}(y)\right|^{2},
$$


where $\hat{\gamma}=\sum_{j} \lambda_{j}\left|g_{j}\right\rangle\left\langle g_{j}\right|$ and $g_{j}=\mathcal{U} f_{j}$. As in (2.3),

$$
\int_{Y} \rho_{\widehat{\gamma}}(y) \mathrm{d} \nu(y)=1 \text {. }
$$

Our final assumption is that $\mathcal{U}$ is bounded from $L^{1}(X)$ to $L^{\infty}(Y)$. This property guarantees that $\mathcal{U}$ has an integral kernel $\mathcal{U}(y, x)$, whose norm is

$$
\begin{aligned}
\infty>\|\mathcal{U}\|_{L^{1} \rightarrow L^{\infty}} & =\operatorname{ess}_{-\sup _{x, y}}|\mathcal{U}(y, x)| \\
& :=\sup \{t:(\mu \times \nu)(\{(x, y):|\mathcal{U}(x, y)|>t\})>0\} .
\end{aligned}
$$

Theorem 2.2. Under the above assumptions, let $\gamma \geq 0$ be an operator in $L^{2}(X)$ with $\operatorname{Tr} \gamma=1$ and such that

$$
\int_{X} \rho_{\gamma}(x) \ln _{+} \rho_{\gamma}(x) \mathrm{d} \mu(x)<\infty \quad \text { and } \int_{Y} \rho_{\widehat{\gamma}}(y) \ln _{+} \rho_{\widehat{\gamma}}(y) \mathrm{d} \nu(y)<\infty,
$$

where $\ln _{+} \rho=\max \{\ln \rho, 0\}$. Then

$$
\begin{aligned}
& -\int_{X} \rho_{\gamma}(x) \ln \rho_{\gamma}(x) \mathrm{d} \mu(x)-\int_{Y} \rho_{\widehat{\gamma}}(y) \ln \rho_{\widehat{\gamma}}(y) \mathrm{d} \nu(y) \\
& \quad \geq-\operatorname{Tr} \gamma \ln \gamma-2 \ln \|\mathcal{U}\|_{L^{1} \rightarrow L^{\infty}} .
\end{aligned}
$$

We illustrate this theorem by some examples.

1. If $X=Y=\mathbb{R}^{d}$ with Lebesgue measure and $\mathcal{U}$ the Fourier transform (i.e., $\mathcal{U}(k, x)=e^{-2 \pi i k \cdot x}$ ), then we recover Theorem 2.1. In this case, $-2 \ln \|\mathcal{U}\|_{L^{1} \rightarrow L^{\infty}}=0$.

2. Let $X=(-L / 2, L / 2)$ with Lebesgue measure, $Y=L^{-1} \mathbb{Z}$ with $L^{-1}$ times counting measure and let $\mathcal{U}$ be the discrete Fourier transform, that is, $\mathcal{U}(k, x)=e^{-2 \pi i k x}$. Then (2.5) holds with $-2 \ln \|\mathcal{U}\|_{L^{1} \rightarrow L^{\infty}}=0$.

3. Let $X=Y=\mathbb{Z} / N \mathbb{Z}=\{0,1, \ldots, N-1\}$ for some $N \in \mathbb{N}$ with counting measure and let $\mathcal{U}(k, n)=N^{-1 / 2} e^{-i 2 \pi k n / N}$. Then (2.5) holds with $-2 \ln \|\mathcal{U}\|_{L^{1} \rightarrow L^{\infty}}=\ln N$.

4. The following is a generalization of Example (3) and is related to $[3,6,8]$. Let $\left(\left|a_{j}\right\rangle\right)_{j}$ and $\left(\left|b_{k}\right\rangle\right)_{k}$ two orthonormal bases in a separable Hilbert space $\mathcal{H}$ and put

$$
c=\sup _{j, k}\left|\left\langle a_{j} \mid b_{k}\right\rangle\right| .
$$

By the Schwarz inequality, $0<c \leq 1$. Let $\gamma \geq 0$ be an operator on $\mathcal{H}$ with $\operatorname{Tr} \gamma=1$. Define

$$
p_{j}:=\left\langle a_{j}|\gamma| a_{j}\right\rangle, \quad q_{k}:=\left\langle b_{k}|\gamma| b_{k}\right\rangle .
$$

Then

$$
-\sum_{j} p_{j} \ln p_{j}-\sum_{k} q_{k} \ln q_{k} \geq-\operatorname{Tr} \gamma \ln \gamma-2 \ln c,
$$

which follows from Theorem 2.2 by noting that, if the change of bases is denoted by $\mathcal{U}$, then $\|\mathcal{U}\|_{L^{1} \rightarrow L^{\infty}}=c$. The weaker inequality without the term $\operatorname{Tr} \gamma \ln \gamma$ on the right side was shown in [8] with a different proof. 
In passing, we note that each of the entropies on the left side of (2.6) is greater than or equal to $-\operatorname{Tr} \gamma \ln \gamma$. This follows from the concavity of $-p \ln p$, the fact (derived from the variational principle) that the sequence $\left(p_{j}\right)$ is majorized by the sequence of eigenvalues of $\gamma$, and Karamata's theorem (see, e.g., [4] or [7, Rem. 4.7]).

\section{Proof of Theorem 2.2}

Our proof is based on the following two well-known lemmas in quantum statistical mechanics (see, e.g., [2,10]).

Lemma 3.1 (Gibbs variational principle). Let $H$ be a self-adjoint operator such that $e^{-H}$ is trace class. Then for any $\gamma \geq 0$ with $\operatorname{Tr} \gamma=1$,

$$
\operatorname{Tr} \gamma H+\operatorname{Tr} \gamma \ln \gamma \geq-\ln \operatorname{Tr} e^{-H}
$$

with equality iff $\gamma=\exp (-H) / \operatorname{Tr} \exp (-H)$.

Lemma 3.2 (Golden-Thompson inequality). For self-adjoint operators $A$ and $B$, bounded from above,

$$
\operatorname{Tr} e^{A+B} \leq \operatorname{Tr} e^{A / 2} e^{B} e^{A / 2} .
$$

Proof of Theorem 2.2. We first assume that the functions $\rho_{\gamma}$ and $\rho_{\widehat{\gamma}}$ are bounded from above and note that

$$
-\int \rho_{\gamma}(x) \ln \rho_{\gamma}(x) \mathrm{d} \mu(x)-\int \rho_{\widehat{\gamma}}(y) \ln \rho_{\widehat{\gamma}}(y) \mathrm{d} \nu(y)=\operatorname{Tr} \gamma H
$$

with the operator $H=-\ln \rho_{\gamma}-\mathcal{U}^{*} \ln \rho_{\widehat{\gamma}} \mathcal{U}$ in $L^{2}(X)$. Here, $\ln \rho_{\gamma}$ and $\ln \rho_{\widehat{\gamma}}$ are considered as multiplication operators, and we used the fact that $\operatorname{Tr}_{L^{2}(X)}$ $\mathcal{U}^{*} A \mathcal{U}=\operatorname{Tr}_{L^{2}(Y)} A$. By Lemmas 3.1 and 3.2,

$$
\begin{aligned}
& -\int \rho_{\gamma}(x) \ln \rho_{\gamma}(x) \mathrm{d} \mu(x)-\int \rho_{\widehat{\gamma}}(y) \ln \rho_{\widehat{\gamma}}(y) \mathrm{d} \nu(y)+\operatorname{Tr} \gamma \ln \gamma \\
& \quad \geq-\ln \operatorname{Tr} e^{-H} \\
& \quad \geq-\ln \operatorname{Tr} \rho_{\gamma}^{1 / 2} \mathcal{U}^{*} \rho_{\widehat{\gamma}} \mathcal{U} \rho_{\gamma}^{1 / 2} .
\end{aligned}
$$

The trace on the right side is the square of the Hilbert-Schmidt norm of the operator $\rho_{\widehat{\gamma}}^{1 / 2} \mathcal{U} \rho_{\gamma}^{1 / 2}$, which has the kernel

$$
\rho_{\widehat{\gamma}}(y)^{1 / 2} \mathcal{U}(y, x) \rho_{\gamma}(x)^{1 / 2} .
$$

Thus,

$$
\begin{aligned}
\operatorname{Tr} \rho_{\gamma}^{1 / 2} \mathcal{U}^{*} \rho_{\widehat{\gamma}} \mathcal{U} \rho_{\gamma}^{1 / 2} & =\iint_{X \times Y} \rho_{\widehat{\gamma}}(y)|\mathcal{U}(y, x)|^{2} \rho_{\gamma}(x) \mathrm{d} \mu(x) \mathrm{d} \nu(y) \\
& \leq\|\mathcal{U}\|_{L^{1} \rightarrow L^{\infty}}^{2} \int_{Y} \rho_{\widehat{\gamma}}(y) \mathrm{d} \nu(y) \int_{X} \rho_{\gamma}(x) \mathrm{d} \mu(x) .
\end{aligned}
$$

By (2.3) and (2.4), this equals $\|\mathcal{U}\|_{L^{1} \rightarrow L^{\infty}}^{2}$, which proves the theorem for bounded $\rho_{\gamma}$ and $\rho_{\widehat{\gamma}}$. 
The general case follows by a simple approximation argument. We consider $H^{(M)}=-\ln \rho_{\gamma}^{(M)}-\mathcal{U}^{*} \ln \rho_{\widehat{\gamma}}^{(M)} \mathcal{U}$ for $M \geq 1$, where $\rho_{\gamma}^{(M)}(x):=$ $\min \left\{\rho_{\gamma}(x), M\right\}$ and similarly for $\rho_{\widehat{\gamma}}^{(M)}(y)$. Then, the previous argument yields

$$
\begin{aligned}
\operatorname{Tr} \gamma H^{(M)}+\operatorname{Tr} \gamma \ln \gamma & \geq-\ln \left(\|\mathcal{U}\|_{L^{1} \rightarrow L^{\infty}}^{2} \int_{Y} \rho_{\widehat{\gamma}}^{(M)}(y) \mathrm{d} \nu(y) \int_{X} \rho_{\gamma}^{(M)}(x) \mathrm{d} \mu(x)\right) \\
& \geq-2 \ln \|\mathcal{U}\|_{L^{1} \rightarrow L^{\infty}}
\end{aligned}
$$

On the other hand, we may assume that the integrals $\int_{X} \rho_{\gamma}(x) \ln _{-} \rho_{\gamma}(x) \mathrm{d} \mu(x)$ and $\int_{Y} \rho_{\widehat{\gamma}}(y) \ln _{-} \rho_{\widehat{\gamma}}(y) \mathrm{d} \nu(y)$ are finite, for otherwise there is nothing to prove. Here $\ln _{-} \rho=\max \{-\ln \rho, 0\}$. In that case, by monotone convergence,

$$
\begin{aligned}
& \lim _{M \rightarrow \infty} \operatorname{Tr} \gamma H^{(M)} \\
& \quad=\lim _{M \rightarrow \infty}\left(-\int \rho_{\gamma}(x) \ln \rho_{\gamma}^{(M)}(x) \mathrm{d} \mu(x)-\int \rho_{\widehat{\gamma}}(y) \ln \rho_{\widehat{\gamma}}^{(M)}(y) \mathrm{d} \nu(y)\right) \\
& =-\int \rho_{\gamma}(x) \ln \rho_{\gamma}(x) \mathrm{d} \mu(x)-\int \rho_{\widehat{\gamma}}(y) \ln \rho_{\widehat{\gamma}}(y) \mathrm{d} \nu(y) .
\end{aligned}
$$

This proves the theorem in the general case.

Note added in proof. After receiving an advance copy of this paper, Michel Rumin was able to extend our results and technique to POVMs in An entropic uncertainty principle for positive operator valued measures, arXiv:1109.5889. Lett. Math. Phys. online first DOI:10.1007/s11005-011-0543-4.

\section{Acknowledgements}

The authors are grateful to M. Rumin for comments on a previous version of this paper.

\section{References}

[1] Beckner, W.: Inequalities in Fourier analysis. Ann. Math. (2) 102(1), 159-182 (1975)

[2] Carlen, E.A.: Trace inequalities and quantum entropy. An introductory course. In: Sims, R., Ueltschi, D. (eds.) Entropy and the Quantum. Contemporary Mathematics, vol. 529, pp. 73-140. American Mathematical Society, Providence (2010)

[3] Deutsch, D.: Uncertainty in quantum measurements. Phys. Rev. Lett. 50, 631$633(1983)$

[4] Hardy, G.H., Littlewood, J.E., Pólya, G.: Inequalities. Cambridge University Press, Cambridge (1952)

[5] Hirschman, I.I. Jr.: A note on entropy. Am. J. Math. 79, 152-156 (1957)

[6] Kraus, K.: Complementary observables and uncertainty relations. Phys. Rev.

D 35, 3070-3075 (1987) 
[7] Lieb, E.H., Seiringer, R.: The Stability of Matter in Quantum Mechanics. Cambridge University Press, Cambridge (2010)

[8] Maassen, H., Uffink, J.B.M.: Generalized entropic uncertainty relations. Phys. Rev. Lett. 60(12), 1103-1106 (1988)

[9] Rumin, M.: Balanced distribution-energy inequalities and related entropy bounds. Duke Math. J. 160(3), 567-597 (2011)

[10] Simon, B.: Trace Ideals and Their Applications, 2nd edn. American Mathematical Soceity, Providence (2005)

Rupert L. Frank

Department of Mathematics

Princeton University

Washington Road

Princeton, NJ 08544

USA

e-mail: rlfrank@math.princeton.edu

Elliott H. Lieb

Departments of Mathematics and Physics

Princeton University

P. O. Box 708, Princeton, NJ 08544

USA

e-mail: lieb@princeton.edu

Communicated by Bernard Nienhuis.

Received: September 14, 2011.

Accepted: February 6, 2012. 